\title{
Der Weg ist das Ziel
}

Zum ,Recht auf Stadt' durch Selbstverwaltung und radikale Demokratie Rezension zu Daniel Mullis

Recht auf die Stadt. Von Selbstverwaltung und radikaler Demokratie.

Die Forderung nach einem ,Recht auf Stadt' stellt sich in den letzten Jahren als übergreifender Bezugspunkt vielfältiger politischer Kämpfe in der ,unternehmerischen Stadt' dar und scheint (manchmal mehr, manchmal weniger) in der Lage zu sein, diverse Interessen und Auseinandersetzungen auf einen gemeinsamen politischen Nenner zu bringen (vgl. Mayer 2011, Harvey 2013). So unterschiedlich die Kämpfe gegen die Vernichtung preiswerten Wohnraums, gegen die Privatisierung öffentlicher Räume, gegen Zwangsräumungen, für die Rechte illegalisierter Menschen, für lokale autonome Zentren oder gegen die Bebauung von Grünflächen auch sein mögen: Gemeinsam ist ihnen zumeist, dass sie von eher marginalisierten Bewohner_innen gegen die Profitinteressen von städtischen Agenturen und Investoren_innen geführt werden. Was das ,Recht auf Stadt ' jedoch konkret bedeutet und wie es durchgesetzt werden kann, wird in aktivistischen und wissenschaftlichen Debatten zwar immer wieder einmal thematisiert, ist aber bislang weitgehend unbeantwortet geblieben. Die auf Henri Lefebvre rekurrierende Deutung, es handele sich hierbei um ein Recht auf Zentralität, städtische Infrastruktur, Wissen, Differenz und Anerkennung (vgl. Holm/Gebhardt 2011: 8), ist dabei vielleicht noch die bekannteste Ausformulierung möglicher Inhalte eines ,Rechts auf Stadt - die jedoch weiterhin viel Interpretationsspielraum lässt, was praktisch mit diesen Forderungen gemeint ist. Noch weniger finden sich explizite Ausführungen zu der Frage, welche Organisationsformen, Praxen und Methoden für die Durchsetzung eines ,Rechts auf Stadt' notwendig sind, oder utopische Überlegungen dazu, wie eine Gesellschaft aussehen müsste, in der das ,Recht auf Stadt' für alle verwirklicht ist.

Viele der in Deutschland entstandenen Recht-auf-Stadt-Bündnisse organisieren sich horizontal und basisdemokratisch. Eine konkrete Bestimmung der Ideen und Konzepte, die eine solche Form der Zusammenarbeit begründen, wird jedoch selten geleistet. Dies verwundert durchaus vor dem Hintergrund der Geschichte linker aktivistischer Praxis, die hierfür viele Anknüpfungspunkte bietet. Insbesondere libertäre oder anarchistische Strömungen könnten einen großen Fundus an 
erprobten Ideen zur horizontalen Organisierung beisteuern - jedoch wurden auch von dieser Seite bisher kaum Verknüpfungen hergestellt (vgl. zu Ausnahmen hierzu die Zeitschrift Direkte Aktion 2013, nbo 2011, Ronneberger 2010).

In seinem Buch Recht auf die Stadt. Von Selbstverwaltung und radikaler Demokratie (2014) nimmt sich Daniel Mullis dieser Fragen an. Er bringt dabei die von Henri Lefebvre stammenden Konzepte, Recht auf Stadt', ,Produktion des Raums' und autogestion (Selbstverwaltung) mit den von Ernesto Laclau und Chantal Mouffe entwickelten Konzepten des ,Politischen' und ,radikaler Demokratie‘ zusammen. Bezugnehmend auf die bekannte Formulierung Lefebvres, Räume würden „produziert“, also sozial hergestellt, fragt Mullis, welche Art der Raumproduktion einer emanzipatorischen politischen Praxis dienlich wäre (65), und stellt dem die Prämisse zur Seite, dass „ein politisches Projekt der Emanzipation nicht nur produziert, sondern immer auch reproduziert werden" muss (144). Er versucht demnach zu klären, in welchem Zusammenhang politische Forderungen nach einem ,Recht auf Stadt' und die organisatorische Praxis der Protestakteure stehen. Dabei betont er explizit die erkenntnistheoretische Nähe der undogmatisch-marxistischen Position Lefebvres sowie der postmarxistisch-poststrukturalistischen Konzepte von Laclau/ Mouffe zu zeitgenössischen anarchistischen Positionen. Insbesondere hinsichtlich „der Betonung der Alltäglichkeit als Ort des Wandels und der sukzessiven ,Revolution““ (9) ergäben sich deutliche Überschneidungen und damit Möglichkeiten der Annäherung.

Dies ist der Ausgangspunkt, von dem aus der Autor beide theoretische Konzeptegegenüberstellt und kritisch diskutiert. Für Mullis bleibt Lefebvres Denken trotz der festgestellten Überschneidungen von essentialistischen Vorstellungen geprägt, die sich etwa darin äußerten, dass er von einer determinierten historischen Entwicklung (ausgedrückt in den „Stufen der Urbanisierung") und einem notwendigen Subjekt der Revolution (der Arbeiterklasse) ausgeht. Dagegen stellten Laclau/Mouffe die Kontingenzjeder historischen Entwicklung und die Bedeutung der (Selbst-)Konstituierung widerständiger Subjekte durch die Formulierung politischer Forderungen in den Vordergrund. Darüber hinaus unterschieden sich die Ebenen der theoretischen Auseinandersetzung der beiden Ansätze: Lefebvre argumentiere stärker auf einer konkreten „realen“ Ebene, das heißt der Ebene der alltäglichen Raumproduktion. Es gehe ihm um „das Urbane“. Laclau/ Mouffe befassten sich dagegen mit „dem Politischen“, sie analysierten stärker die erkenntnistheoretischen Hintergründe politischer Aushandlungen in dekonstruktivistischer Absicht. Mullis Anliegen ist es dabei einerseits, mithilfe der Argumentation von Laclau/Mouffe Lefebvres Essentialismen infrage zu stellen, andererseits das Konzept der, radikalen Demokratie‘ von Laclau/Mouffe durch die Konfrontation mit Lefebvre „konkret“ und für die alltägliche politische Praxis nutzbar zu machen.

Das Buch beginnt mit einer sehr anregenden Zusammenstellung aktueller städtischer Konflikte und Protestbewegungen, von Brasilien über die USA bis hin zur Türkei, in denen das ,Recht auf Stadt' für das Selbstverständnis der Protestierenden eine zentrale Rolle spielt(e). Besonderes Augenmerk finden dabei die Besetzungen zentraler städtischer 
Plätze, die als „Streik der Stadt“ (19) interpretiert werden. Diesem praxisnahen Einstieg folgt zunächst eine getrennte Analyse der Ansätze von Lefebvre und Laclau/Mouffe, die dann in einem zweiten Schritt auf ihre Gegensätze und Gemeinsamkeiten hin untersucht werden. Hierbei fällt auf, dass der Autor die Konzepte Lefebvres ausführlicher und leser_innenfreundlicher aufbereitet, indem er zum Beispiel eine biografisch-historische Kontextualisierung seines Werks vornimmt. In Kapitel 3 widmet er sich dem in der politischen Praxis viel genutzten Konzept ,Recht auf Stadt' und stellt es in einen Zusammenhang mit Lefebvres Ausführungen zur ,Produktion des Raums` und zu autogestion. Denn die Frage, wie ein Recht auf Stadt praktisch umgesetzt werden kann - also welcher ,Weg'von der Bewegung eingeschlagen werden sollte -, hängt nach Mullis essenziell mit der Frage zusammen, welche Form der Raumproduktion von den beteiligten Akteuren gewählt wird beziehungsweise gewählt werden kann. Dabei macht er deutlich, dass unter kapitalistischen Verhältnissen ein ,Recht auf Stadt' solange eine Illusion bleiben muss, bis es widerständigen Praktiken gelingt, die Verhältnisse tatsächlich (im Sinne eines Brechens bestehender kapitalistischer Hegemonie) infrage zu stellen. Zudem muss in diesen Praxen - also in ihrer Organisationsform - schon gleichzeitig die Vision von einem grundlegend anderen und egalitären gesellschaftlichen Zusammenleben zum Ausdruck kommen. Insbesondere die beiden Unterkapitel „Recht auf die Stadt“ (3.3) und „Selbstverwaltung und emanzipatorische Praxis“ (3.4) sind eine hilfreiche theoretische Reflexion darüber, warum eine solche horizontale organisatorische Praxis erforderlich ist, und leisten damit einen Beitrag, die angesprochene ,Theorie-PraxisLücke، vieler Recht-auf-Stadt-Netzwerke zu schließen.

Die poststrukturalistischen Konzepte von Laclau/Mouffe werden in Kapitel 4 dargestellt. Diese machen Kontingenz zu einer grundlegenden Kategorie ihres Denkens, wodurch es ihnen nach Mullis - im Gegensatz zu Lefebvre - gelänge, die prinzipielle Offenheit historischer Entwicklung nicht nur zu postulieren, sondern auch konsequent in ihre theoretischen Überlegungen einzubeziehen. Die Konzepte der ,politischen Differenz und der ,radikalen Demokratie، seien darüber hinaus auch deswegen hilfreich, da sie sowohl auf die Frage der Wiederbelebung politischer Konflikte in einer scheinbar befriedeten kapitalistischen Wohlstandsgesellschaft nach dem Kalten Krieg eingehen würden als auch auf die Möglichkeit einer radikal-egalitären Demokratie. Meines Erachtens ist der Ansatz, Lefebvre mit Laclau/ Mouffe zusammenzudenken, auch deshalb innovativ, weil in Debatten und Arbeiten der kritischen Geographie der ,Klassiker ${ }^{6}$ Lefebvre bislang kaum mit deren Ideen konfrontiert worden ist - dieses Vorgehen aber durchaus das Potenzial bietet, bestehende theoretische Gräben zwischen materialistischen und poststrukturalistischen Ansätzen weiter zu überbrücken.

Die Teile des Buchs, in der sich der Autor mit Laclau/Mouffe auseinandersetzt, sind aufgrund der hohen Komplexität ihrer Analysen und der an manchen Stellen doch etwas holprigen Darstellung stellenweise etwas mühsam zu lesen. Besonders für Leser_innen ohne profunde Kenntnisse linker sozialwissenschaftlicher Debatten dürften sie eher schwer zugänglich sein. Mullis gelingt es nicht wirklich, die Konzepte der ,politischen 


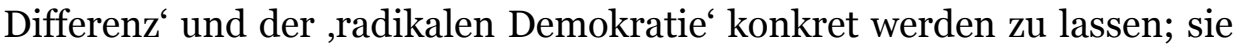
verbleiben vielmehr auf einer hohen Abstraktionsebene.

In den Kapiteln 5 und 6 führt der Autor dann die verschiedenen theoretischen Diskussionen zusammen und versucht diese in eine „Sprache der Praxis“ (29) zu übersetzen. Eine Zusammenführung der Abstraktionsebenen des Urbanen und des Politischen ergibt sich für Mullis, indem das Urbane als „eine Bezeichnung für eine soziale und räumlich Strukturiertheit, die das Politische ermöglicht und unterstützt“ (140), gedacht wird. Wird das Urbane dabei als je temporäres Resultat gleichermaßen andauernder und kontingenter (alltäglicher) Auseinandersetzungen betrachtet, wird deutlich, warum die Frage nach dem ,Wie ' widerständiger Raumproduktion eine entscheidende ist. Dabei sieht Mullis die Erkenntnis, dass „kein historischer Zwang der Urbanisierung, keine basale Eigenschaft des Subjekts, kein historisch begründeter Klassenkampf [...] dem Recht auf die Stadt zu Hilfe eilen“ wird (153), als wichtige Erweiterung der Lefebvre'schen Argumentation an. Aus dieser Perspektive ginge es für die politische Bewegung darum, „, sich in der Praxis immer zu fragen, welche Räume produziert werden, welche Ausschlüsse [dabei] hergestellt und warum diese hergestellt werden" (154) - und wie sie überwunden werden können.

So wird vom Autor zwar ausdrücklich auf das Verhältnis von Theorie und Praxis eingegangen, dennoch will die Verbindung mit aktueller politischer Praxis nicht so recht gelingen: $\mathrm{Zu}$ weit weggerückt ist in den Ausführungen des Buches das alltägliche Leben mit seinen Kämpfen - das doch als Ausgangspunkt des Erkenntnisinteresses benannt wurde. Dabei könnte eine aus der Praxis entwickelte Theoriediskussion zur Frage, wie die Organisation des politischen Alltags einer möglichen anderen, nicht profitorientierten, sondern solidarischen ,Produktion des Raums aussehen könnte, durchaus wichtig sein, auch für die Perspektiven und Auseinandersetzungen in zahlreichen städtischen Bewegungen und Bündnissen. An empirischen Beispielen mangelt es gegenwärtig sicherlich nicht: Während in Hamburg-St. Pauli mit dem Park Fiction, der Wunschproduktion zur Rindermarkthalle, mit Stadtteilversammlungen und aktuell der Planbude zur Gestaltung des Neubaus der umkämpften Esso-Häuser in den letzten Jahren vielfach von Recht-auf-Stadt-Aktivist_ innen mit basisdemokratisch orientierter, partizipativer Planung experimentiert wurde (vgl. Füllner/Templin 2011, Planbude 2014), ist in Freiburg eine Initiative für einen „Stadtteil von unten“ entstanden (vgl. BISS o.J.). In Frankfurt am Main ringt gerade eine Gruppe von Stadtteilaktivist_innen um den Zuschlag für ein großangelegtes ,solidarisches Wohnprojekt mitten auf dem Campus Bockenheim (Philosophicum o.J.) - ganz abgesehen von den unzähligen Mieter_inneninitiativen, die mal mehr, mal weniger gut vernetzt um bezahlbare Mieten und ihren Verbleib im Stadtteil kämpfen (Schulte-Westenberg/Coers 2014).

Das Buch von Mullis beantwortet also weniger praktische Fragen der Selbstverwaltung und Demokratie, sondern fordert auf, sich in zukünftigen Auseinandersetzungen um ein ,Recht auf Stadt' stärker der organisatorischen Frage zu widmen; das heißt der Frage, wie sich die kapitalistische Waren- und Kontrollgesellschaft von unten her durch eine permanente 
Politisierung des Alltags und einer herrschaftsfreien Selbstverwaltung verändern lässt. Dazu bietet es ein komplexes und vielversprechendes theoretisches Rüstzeug an - das auf seine empirische und praktische Überprüfung wartet.

\section{Autor innen}

Joscha Metzger; Arbeitsschwerpunkte: urbane Konflikte und Proteste, Stadtentwicklung und Wohnen

joscha.metzger@uni-hamburg.de

\section{Literatur}

BISS (o.J.): BasisInitiative Stadtquartier Schildacker. Ein sozialer, ökologischer, selbstverwalteter Stadtteil von Unten! http://biss-freiburg.de (letzter Zugriff am 20.8.2014).

Direkte Aktion (2013): Die Stadt und die Fabrik (Ausgabe vom November/Dezember 2013). https://www.direkteaktion.org/220/da_220.pdf (letzter Zugriff am 20.8.2014).

Füllner, Jonas / Templin, David (2011): Stadtplanung von unten. Die „Recht auf Stadt“Bewegung in Hamburg. In: Initiativen für ein Recht auf Stadt. Theorie und Praxis städtischer Aneignung. Hamburg: VSA-Verlag, 79-104.

Harvey, David (2013): Rebellische Städte. Vom Recht auf Stadt zur urbanen Revolution. Berlin: Suhrkamp Verlag.

Holm, Andrej / Gebhardt, Dirk (2011): Initiativen für ein Recht auf Stadt. In: dies. (Hg.), Initiativen für ein Recht auf Stadt. Theorie und Praxis städtischer Aneignung. Hamburg: VSA-Verlag, 7-23.

Mayer, Margit (2011): Recht auf die Stadt-Bewegungen in historisch und räumlich vergleichender Perspektive. In: Andrej Holm / Dirk Gebhardt (Hg.), Initiativen für ein Recht auf Stadt. Theorie und Praxis städtischer Aneignung. Hamburg: VSA-Verlag, 53-77.

nbo (2011): Recht auf Stadt - mehr als eine griffige Parole? http://wiki.rechtaufstadt.net/ index.php/Recht_auf_Stadt_-_mehr_als_eine_griffige_Parole\%3F (letzter Zugriff 21.8.2014).

Planbude (2014): Wir nennen es Planbude - Konzept. http://planbude.de/ (letzter Zugriff am 20.8.2014).

Philosophicum (o.J.): Leben im Philosophicum. Selbstbestimmt und solidarisch. http:// philosophicum.org/startseite/leben-im-philosophicum (letzter Zugriff am 20.8.2014).

Ronneberger, Klaus (2010): Die Frage der Autogestion. Henri Lefebvre, Selbstverwaltung und Partizipation. In: analyse \& kritik 550/2010, 32-34.

Schulte-Westenberg, Gertrud / Coers, Matthias (2014): Mietrebellen. Widerstand gegen den Ausverkauf der Stadt (Dokumentarfilm). http://mietrebellen.de/film (letzter Zugriff am 20.8.2014) 